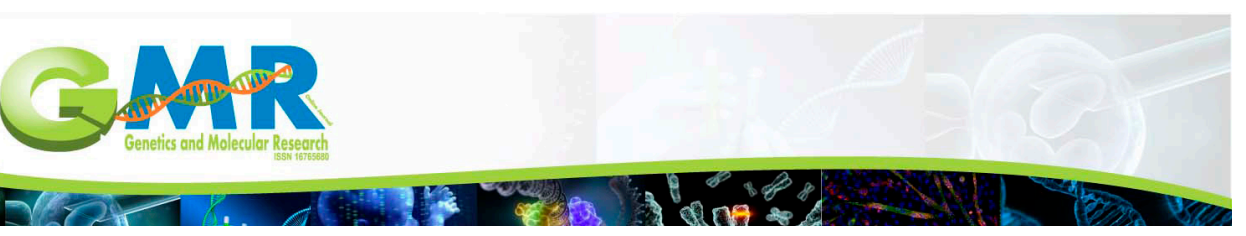

\title{
Cloning and functional analysis of goat SWEET1
}

\author{
L.Q. Zhu*, Z.K. Bao*, W.W. Hu, J. Lin, Q. Yang and Q.H. Yu \\ Veterinary College, Nanjing Agricultural University, Weigang 1, Nanjing, Jiangsu, \\ China \\ *These authors contributed equally to this study. \\ Corresponding author: Q.H. Yu \\ E-mail: yuqinghua1981@163.com
}

Genet. Mol. Res. 14 (4): 17124-17133 (2015)

Received September 18, 2015

Accepted November 11, 2015

Published December 16, 2015

DOI http://dx.doi.org/10.4238/2015.December.16.12

ABSTRACT. SWEETs are a recently discovered class of sugar transporters that mediate glucose uptake in the intestine and mammary glands. Our objectives were to clone goat SWEET1 and conduct a functional analysis of its effect on glucose efflux in goat mammary gland epithelial cells. We cloned and sequenced the goat SWEET1 gene from goat mammary glands, then conducted an analysis of the structure of goat SWEET1, including a prediction of the transmembrane helices and potential $\mathrm{N}$-glycosylation sites. To investigate the biological function of goat SWEET1, we also generated goat SWEET1-transfected goat mammary gland epithelial cells using the eukaryotic expression vector pcDNA3.1-gSWEET1. Goat SWEET1 overexpression can reduce glucose absorption in mammary gland epithelial cells with increasing expression of GLUT1, GLUT4, and GLUT12, which may be attributed to glucose efflux arising from the leading role played by goat SWEET1. This study will improve our understanding of the glucose balance in mammary glands and the level of glucose in milk.

Key words: Goat; SWEET1; Mammary gland epithelial cells; Glucose transporters 


\section{INTRODUCTION}

Cellular uptake and efflux of sugars across the plasma membrane is one of the most important processes for growth and development, and is always mediated by membrane-associated carrier proteins: $\mathrm{Na}^{+} /$glucose co-transporters (SGLTs) and facilitative glucose transporters (GLUTs) (Walmsley et al., 1998; Scheepers et al., 2004). Recently, a new class of glucose transporters, named SWEETs, has been identified in Arabidopsis thaliana (Chen et al., 2010; Chen et al., 2012). The SWEET family is conserved in bacteria, plants, and humans, and is not structurally related to either the GLUT or SGLT family (Baker et al., 2012; Saudek, 2012; Chardon et al., 2013). SWEET1 participates in glucose efflux activity in A. thaliana (Chen et al., 2010). However, the physiological role of SWEETs in mammals remains uncertain (Zhao, 2014).

In this study, we cloned the goat SWEET1 gene (gSWEET1) coding DNA sequence (CDS) region and analyzed the similarities in the SWEET1 gene between different species. Moreover, we also evaluated the biological function of gSWEET1 in glucose efflux activity in goat mammary gland epithelial (GMGE) cells.

\section{MATERIAL AND METHODS}

\section{Cloning gSWEET1 and construction of the expression vector}

Total RNA was isolated using a GeneJET'тм RNA Purification Kit (Fermentas, Sweden, EU) from mammary gland tissue obtained from Saanen dairy goats (Capra hircus). The total RNA was treated with RNase-free DNase I (Fermentas) and used to synthesize the first-strand complementary DNA (cDNA). The sequences of the primer oligonucleotides used in this study are listed in Table 1. The partial gSWEET1 gene was amplified from the first-stand cDNA and cloned into the pJET1.2 ${ }^{\mathrm{TM}}$ vector (Fermentas) for sequencing. The cDNA was amplified using Phusion ${ }^{\mathrm{TM}}$ Hot Start High-Fidelity DNA Polymerase (Fermentas) with primers gSWEET1-F and gSWEET1-R. The 3' sequence had a frameshift mutation compared with the published sequence (XM_005677408.1). The CDS region of gSWEET1 (681 bp) was amplified from the partial gSWEET1 (Figure 1A). The gSWEET1 gene was cloned into the pcDNA3.1 (+) vector to construct pcDNA3.1-gSWEET1 and verified (Figure $1 \mathrm{~B}$ and $1 \mathrm{C}$ ).

\section{Sequence analysis of goat SWEET1}

The cDNA sequence was analyzed using CLUSTAL W2.1 (http://www.ebi.ac.uk/Tools/ msa/clustalw2/), the National Center for Biotechnology Information BLAST site (http://blast.ncbi. nlm.nih.gov/Blast.cgi), and the TMHMM Server v. 2.0 program (http://www.cbs.dtu.dk/services/TMHMM/). The N-glycosylation sites of gSWEET1 were predicted with NetNGlyc 1.0 Server (www. cbs.dtu.dk/services/NetNGlyc). The multiple alignment of the 13 SWEET1 protein sequences was conducted with CLUSTAL W2.1 and the phylogenetic analysis was performed using the neighborjoining method with the Poisson correction (Tamura et al., 2011). 
Table 1. Sequences of oligonucleotide primers used for polymerase chain reaction (PCR) and reverse transcription quantitative PCR (RT-qPCR).

\begin{tabular}{lllc}
\hline Primer name & Type & Sequence & Product length (bp) \\
\hline gSWEET1-F1 & Forward & CTCGAGATGGAGGCGGGCGGATTGGCCGACTC & 681 \\
gSWEET1-R1 & Reverse & CTCGAGTCAGGTTTGTAGAAGCTGATAGTTCCTGTC & 693 \\
gSWEET1-F2 & Forward & CTCGAGATGGAGGCGGGGGATTGGC & 134 \\
gSWEET1-R2 & Reverse & CTCGAGTCAAGTGAACTCCCTCAGGTTTGTAGAAGC & \\
GLUT1-F & Forward & CGCTTCCTGCTCATTAAC & 111 \\
GLUT1-R & Reverse & GCTCTCCTCCTTCATCTC & \\
GLUT4-F & Forward & TGCTCATTCTTGGACGGTTCT \\
GLUT4-R & Reverse & CTAGCACCTGGGCGATTAGG & \\
GLUT12-F & Forward & TCACTGGCCAGCCAAACATA \\
GLUT12-R & Reverse & AACGAAGCTGCCATCACAGA \\
$\beta-$-actin-F & Forward & CACCACACCTTCTACAAC \\
$\beta-$-actin-R & Reverse & TCTGGGTCATCTTCTCAC & 106 \\
AKT2-F & Forward & TGTCATCAAAGAAGGCTGGC \\
AKT2-R & Reverse & AAGGTATTGGGCCGAGGTCT \\
IGF2-F & Forward & GCATCGTGGAGGGTTTGC \\
IGF2-R & Reverse & CACGGGGTATGCTGTGAAGT \\
EEF1A1-F & Forward & CATCCCAGGCTGACTGTGC \\
EEF1A1-R & Reverse & TGTAAGCCAAAAGGGCATGC \\
EEF2-F & Forward & GGGCTGGTGTCTACTGGCC \\
EEF2-R & Reverse & TCAGGATTGTCCTCTGGATC \\
gSWEET1-F & Forward & GTGCTCCTTCAGACTACA \\
gSWEET1-R & Reverse & GTGGTGAGAGGTACATACT & 149 \\
\hline
\end{tabular}

A

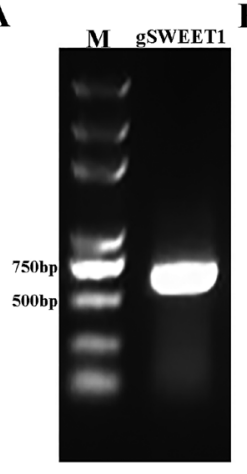

B

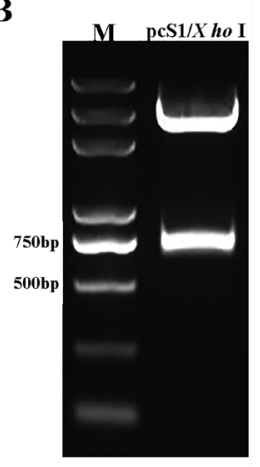

C

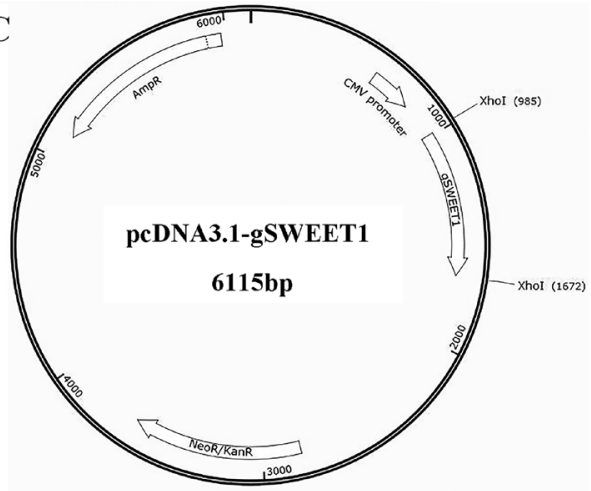

Figure 1. Construction and identification of pcDNA3.1-gSWEET1. A. Polymerase chain reaction (PCR) analysis with primers gSWEET1-F/gSWEET1-R. Lane $M=$ Trans2KTM plus DNA marker, gSWEET1 681 bp PCR production of goat SWEET1. B. Identification of pcDNA3.1-gSWEET1 by digestion with Xhol. Lane $M=$ Trans2KTM plus DNA marker, pcS1/Xhol gSWEET1 inserted into pcDNA3.1(+) and digested with Xhol. C. pcDNA3.1-gSWEET1 vector construction diagram.

\section{GMGE cell isolation and culture}

GMGE cells were isolated from the mammary gland tissue of Saanen dairy goats and cultured with the basal growth medium Dulbecco's modified Eagle's medium (DMEM)/F12 plus $10 \%$ fetal bovine serum. Progesterone $\left(5 \mathrm{mg} / \mathrm{mL}\right.$ ) (ProSpec, ISR, AS), $10^{-7} \mathrm{M}$ hydrocortisone (R\&D, $\mathrm{CA}, \mathrm{USA}$ ), and $10 \mathrm{ng} / \mathrm{mL}$ ovine epithelial growth factor (ProSpec) were added to the basal growth medium to stimulate the growth of GMGE cells. 


\section{Transfection of pcDNA3.1-gSWEET1 into GMGE cells}

When the GMGE cells had grown to $80 \%$ confluence, we discarded the serum and continued to culture the cells for $48 \mathrm{~h}$. Lipofectamine ${ }^{\mathrm{TM}} 2000(60 \mu \mathrm{L})$ (Invitrogen, Carlsbad, CA, USA) containing $24 \mu \mathrm{g}$ pcDNA3.1-gSWEET1 was added to $15 \mathrm{~mL}$ DMEM/F12 without fetal bovine serum, then cultured for $6 \mathrm{~h}$. The medium was then discarded and the cells were cultured for 2 weeks with the culture medium mentioned above plus G418 (600 $\mu \mathrm{g} / \mathrm{mL})$ (Invitrogen) until stable gSWEET1-transfected GMGE (ST1-GMGE) cells were ready for selection.

\section{Glucose detection in the cell culture medium}

Culture medium was collected from the ST1-GMGE cells and GMGE cells, which were both cultured in 6-well plates at the same density. We isolated cell protein using radioimmunoprecipitation assay lysis buffer and detected the protein concentration using the BCA method. The glucose level in the culture medium was determined using a Glucose Assay Kit II (BioVision, Tucson, AZ, USA).

\section{The effect of the overexpression of gSWEET1}

Total RNA was extracted from the ST1-GMGE and GMGE cells using a GeneJETTM RNA Purification Kit (Fermentas). To determine the gene expression profiles, $2 \mu \mathrm{L}$ cDNA was amplified in a $20 \mu \mathrm{L}$ reaction mix containing $10 \mu \mathrm{L}$ of SYBR Premix ${ }^{\mathrm{TM}}$ Ex Taq (TaKaRa, Dalian, China), $0.4 \mu \mathrm{L}$ ROX dye II, and specific primers (Table 1) with an ABI 7500 instrument. The data are reported as values normalized to the housekeeping gene (the gene encoding $\beta$-actin) to account for repeated measures. The differences between the means at each stage of development were determined.

\section{Statistical analysis}

A statistical analysis was performed using one-way analysis of variance. The comparison of the means was performed, and the differences were considered significant if $P<0.05$.

\section{RESULTS}

\section{Cloning of gSWEET1}

A partial 681 bp gSWEET1 gene sequence containing the CDS region was successfully amplified (Figure 1A), and the open reading frame analysis suggested that the gSWEET1 was composed of 226 amino acids. The DNA sequence of gSWEET1 (GenBank: KP997221) is 99\% identical to the goat sequence published in NCBI (XM-005677408.1). The phylogenetic analysis of protein sequences showed that gSWEET1 protein was most similar to the sheep and bovine proteins (Figure 2). Moreover, the DNA sequence of gSWEET1 was 97, 85, and 83\% identical to the sequences of bovine (XM-003585826.3), human (NM-018845.3), and mouse (NM-009057) SWEET1, respectively. The deduced amino acid sequence of gSWEET1 was 97,83 , and $80 \%$ identical to the sequences of bovine (XP-003581980.1), human (NP-061333.2), and mouse (NP033083.2) SWEET1, respectively (Figure 3). 


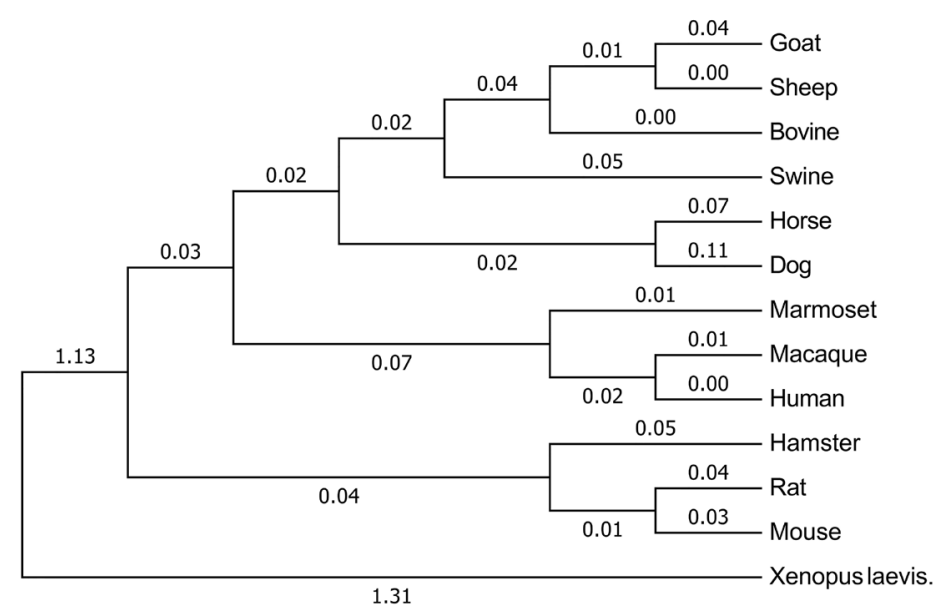

Figure 2. Phylogenetic analysis of goat SWEET1. A phylogenetic tree of protein sequences from goat and other vertebrates. GenBank accession numbers are as follows: SWEET1 sheep (XM_004002573.1), SWEET1 bovine (XM_003585826.3), SWEET1 swine (XM_003125719.2), SWEET1 horse (XM_001498337.2), SWEET1 dog (XM_003434894.2), SWEET1 marmoset (XM_002760075.3), SWEET1 macaque (XM_005541604.1), SWEET1 human (NM_018845.3), SWEET1 hamster (XM_005080168.1), SWEET1 rat (NM_001106445.1), SWEET1 mouse (XM_006501155.1), and SWEET1 Xenopus laevis (NM_001091035.1). The scale bar represents the number of substitutions per amino acid site.

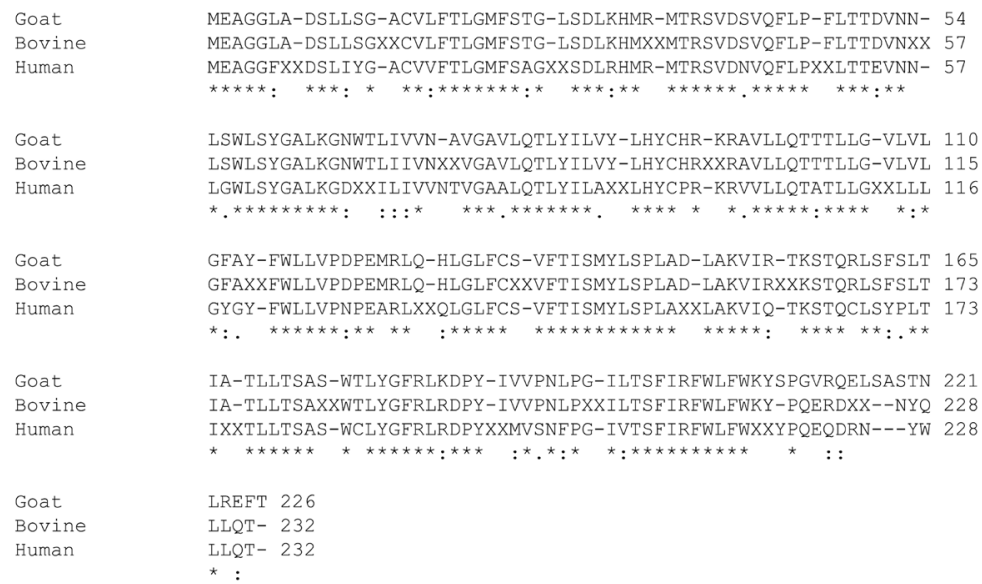

Figure 3. Multiple sequence alignment of the deduced amino acid sequence of goat SWEET1 with other species. The alignments were performed with the CLUSTAL W2.1 program. An asterisk indicates the positions which have a single, fully conserved residue. A colon indicates conservation between groups of strongly similar properties scoring $>0.5$ in the Gonnet PAM 250 matrix. A period indicates conservation between groups of weakly similar properties scoring $\leq 0.5$ in the Gonnet PAM 250 matrix.

\section{Sequence analysis of gSWEET1}

The prediction of gSWEET1 with TMHMM Server v. 2.0 (Figure 4A) revealed that the gSWEET1 had seven transmembrane (TM) helices, nearly identical to bovine and human SWEET1 
(Figure 4B and 4C). The gSWEET1 has two conserved MtN3/saliva motifs embedded in the tandem 3-TM repeat unit, which are connected with a central TM helix. By using the NetNGlyc 1.0 Server (www.cbs.dtu.dk/services/NetNGlyc), four potential N-glycosylation sites were predicted at amino acids 53-56 (NNLS) in the first TM-domain, at 66-69 (NWTL), at 73-76 (NAVG) in the second TM-domain, and at 191-194 (NLPG) in the seventh TM-domain (Figure 5).

A

TMHMM posterior probabilities for Goat

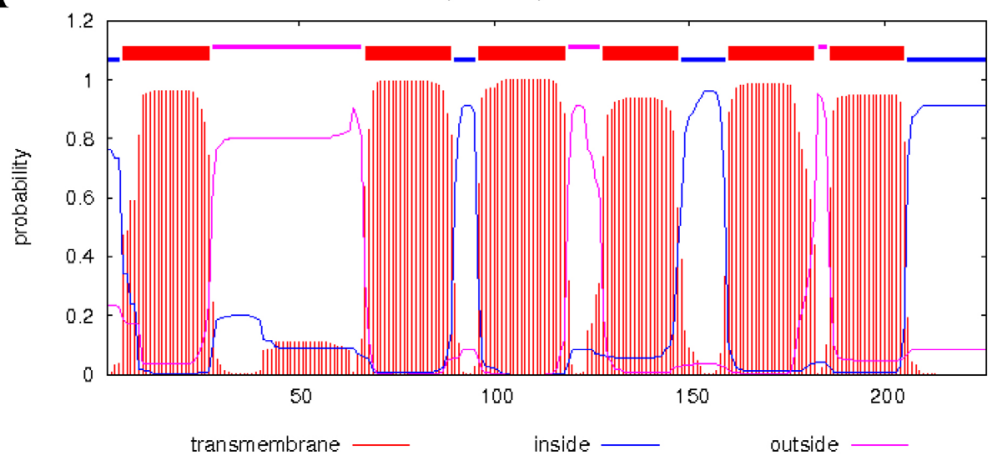

B

TMHMM posterior probabilities for Bovine

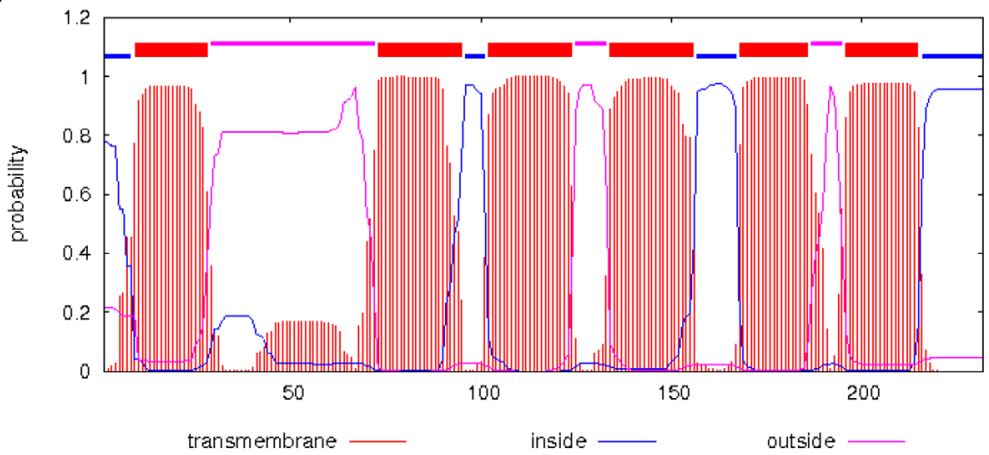

C

TMHMM posterior probabilities for Human

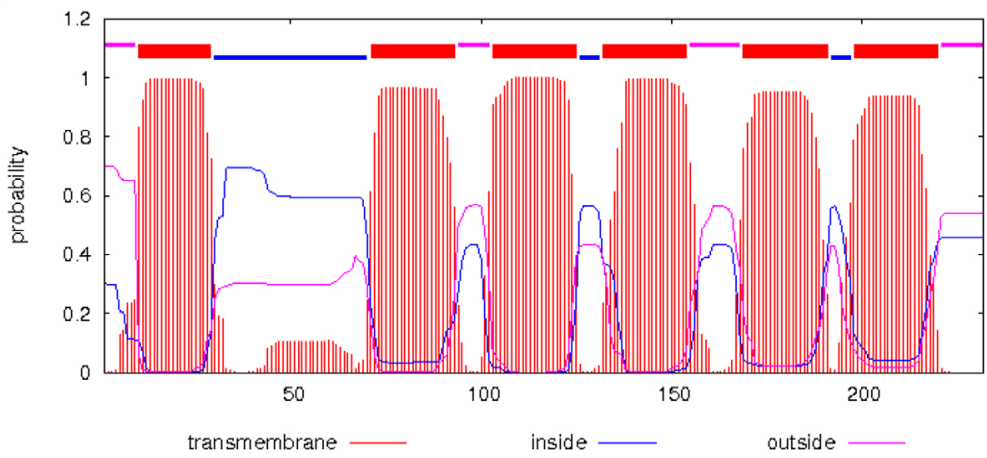

Figure 4. Transmembrane helices prediction of goat SWEET1. Red lines indicate transmembrane regions, blue lines indicate intracellular regions, and pink lines indicate extracellular regions. The vertical axes indicate the probability of transmembrane helices, and the horizontal axes indicate amino acid sequences. A. Goat SWEET1 amino acid prediction. B. Bovine SWEET1 amino acid prediction. C. Human SWEET1 amino acid prediction. 


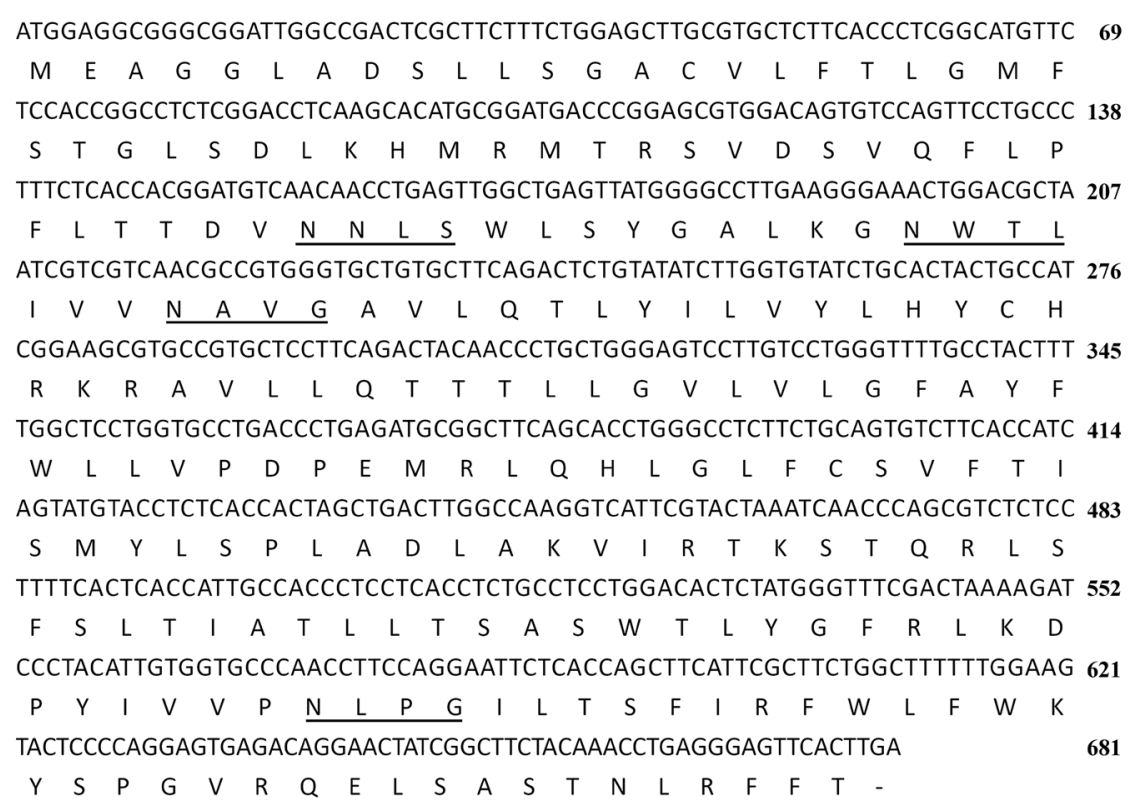

Figure 5. The coding DNA sequence (CDS) region of CDNA and deduced amino acid sequence of gSWEET1. The odd rows are sequences of CDNA, and the even rows are the sequences of the amino acids. Four potential $\mathrm{N}$-glycosylation sites are at amino acids 53-56 (NNLS), 66-69 (NWTL), 73-76 (NAVG), and 191-194 (NLPG).

\section{The mRNA expression of gSWEET1 and glucose transporters in ST1-GMGE cells}

The mRNA expression of gSWEET1 in the ST1-GMGE cells was significantly increased compared with the GMGE cells $(P<0.05)$ (Figure 6A). With the overexpression of gSWEET1, the mRNA expression levels of GLUT1 and GLUT12 in the ST1-GMGE cells were both increased by over 5-fold compared with the GMGE cells (Figure 6B).
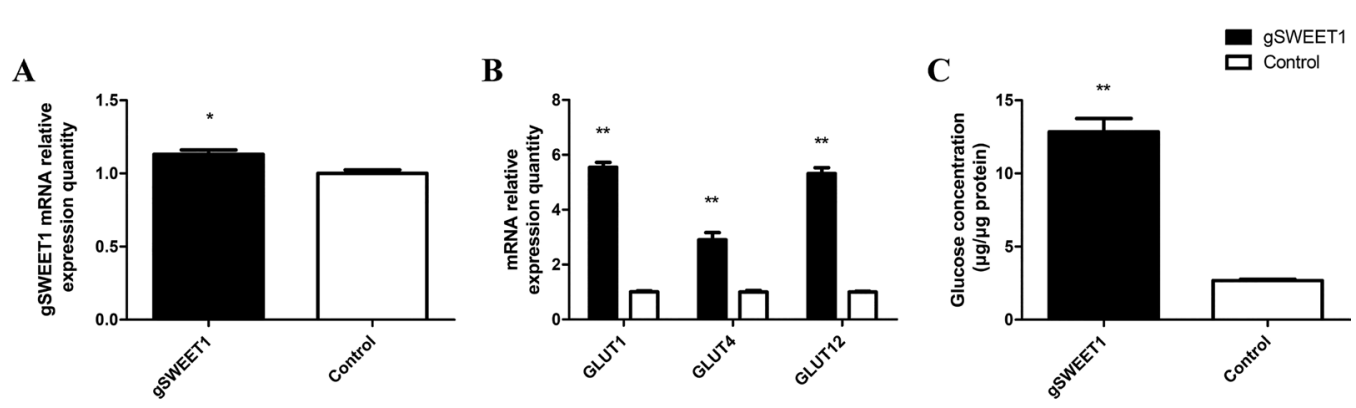

Figure 6. Goat SWEET1 (A) and GLUTs (B) mRNA levels in ST1-GMGE and GMGE cells (GMGE cells are goat mammary gland epithelial cells). In GMGE cells, the expression levels of SWEET1 and GLUTs were significantly affected by transfection of pcDNA3.1-gSWEET1. (C) glucose efflux in GMGE cells. Control means the GMGE cellstransfected pcDNA3.1 (+) vector, gSWEET1 means the stable ST1-GMGE cells. The vertical axes indicate the mean glucose efflux and total protein radio. The horizontal axes indicate the different groups. All data are expressed as mean $\pm \mathrm{SE}(\mathrm{N}=3) .{ }^{*} \mathrm{P}<0.05,{ }^{* *} \mathrm{P}<0.01$, compared with GMGE cells. 


\section{mRNA expression of genes related to the glycometabolism signaling pathway}

The overexpression of gSWEET1 significantly reduced the mRNA expression levels of the elongation factors EEF1A1 and EEF2 in ST1-GMGE cells $(P<0.01)$ (Figure 7B). We also found that the mRNA expression levels of two insulin signaling pathway-related genes, insulin-like growth factor-2 (IGF2) and AKT2, increased significantly $(P<0.01)$ (Figure 7A).

A

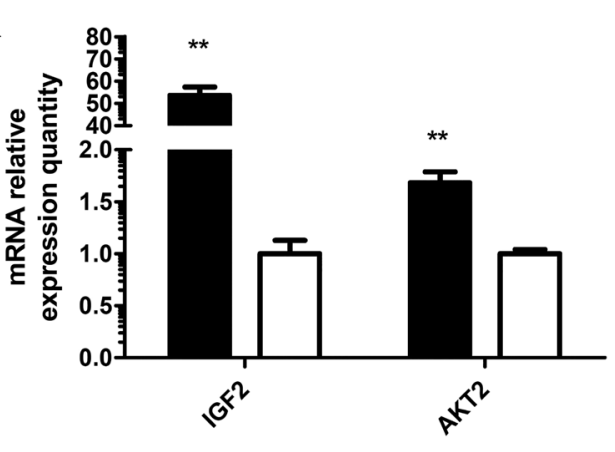

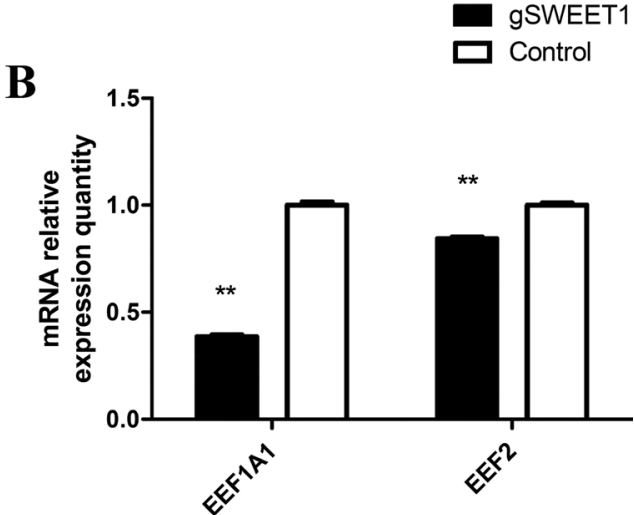

Figure 7. Transcription elongation factor and insulin signaling pathway genes mRNA levels in ST1-GMGE cells and GMGE cells (GMGE cells are goat mammary gland epithelial cells). Expression levels of IGF-2, AKT-2, EEF1A1, and EEF2 were significantly affected by the transfection of pcDNA3.1-gSWEET1 $(P<0.01)$. Data are expressed as mean $\pm \mathrm{SE}(\mathrm{N}=9)$. ${ }^{*} \mathrm{P}<0.05 ;{ }^{* *} \mathrm{P}<0.01$, compared with GMGE cells. Control = GMGE cells; gSWEET1 = ST1-GMGE cells.

\section{Detection of glucose consumption in ST1-GMGE cells}

In order to evaluate the biological function of the gSWEET1 gene, glucose consumption was assessed. The glucose concentration in the cell medium of the ST1-GMGE cells was significantly higher (by nearly 60\%; P < 0.01) than in that of the GMEM cells, which means that the consumption of glucose in the ST1-GMGE cells was lower than in the GMGE cells (Figure 6C).

\section{DISCUSSION}

In this study, we cloned and sequenced partial gSWEET1 cDNA, which closely resembles that of sheep (96\%) and cattle (95\%). The gSWEET1 had seven transmembrane domains with four potential N-glycosylation sites, which is highly homologous to bovine and human SWEET1. Furthermore, the phylogenetic tree analysis confirmed their genetic relationships. However, this is different from GLUTs and SGLTs, which have 12 and 14 domains, respectively (Zhao and Keating, 2007; De Zutter et al., 2013). The structure of gSWEET1 is consistent with that found in $A$. thaliana (Xuan et al., 2013), indicating high conservation in the different species.

The mRNA expression of gSWEET1 in ST1-GMGE cells was significantly increased, which in turn demonstrated successful transfection. Accompanying the gSWEET1 overexpression, the mRNA expression levels of GLUT1, GLUT4, and GLUT12 were also significantly increased by different degrees. Considering the efflux activity of gSWEET1 in $A$. thaliana, we speculated that gSWEET1 and the GLUTs cooperated in the modulation of glucose balance in mammary gland 
cells (Chen et al., 2010). Along with the glucose efflux activity caused by gSWEET1 overexpression, cells need to absorb more glucose to balance the glucose concentration in the cytoplasm, which may be the cause of the high expression of GLUT1, GLUT4, and GLUT12 (Macheda et al., 2003; Thorens and Mueckler, 2010). This is also confirmed by the increase of glucose concentration in the medium of ST1-GMGE cells with overexpression of gSWEET1.

IGF2 and AKT2 can take part in the insulin signaling pathway and promote energy utilization (Nozaki et al., 2013; Bach, 2015). The increasing expression of AKT2 could modulate the insulin signaling pathway and induce the translocation of insulin-sensitive GLUT4 from intracellular pools to the plasma membrane, thereby stimulating the absorption of more glucose from blood to balance the glucose efflux activity caused by gSWEET1 (Stuart et al., 2009; Leto and Saltiel, 2012). Low glucose concentration in the cytoplasm resulting from overexpression of gSWEET1 would then inhibit transcription. This is consistent with the decreasing expression levels of translation elongation factors EEF2 and EEF1A1 (Bionaz and Loor, 2011).

\section{Conflicts of interest}

The authors declare no conflict of interest.

\section{ACKNOWLEDGMENTS}

Research supported by grants from the National Animal Transgenic Breeding Grand Project (\#2014ZX08008-004) and a project funded by the Priority Academic Program Development of Jiangsu Higher Education Institutions.

\section{REFERENCES}

Bach LA (2015). Endothelial cells and the IGF system. J. Mol. Endocrinol. 54: R1-R13.

Baker RF, Leach KA and Braun DM (2012). SWEET as sugar: new sucrose effluxers in plants. Mol. Plant 5: 766-768.

Bionaz M and Loor JJ (2011). Gene networks driving bovine mammary protein synthesis during the lactation cycle. Bioinform. Biol. Insights 5: 83-98.

Chardon F, Bedu M, Calenge F, Klemens PA, et al. (2013). Leaf fructose content is controlled by the vacuolar transporter SWEET17 in Arabidopsis. Curr. Biol. 23: 697-702.

Chen LQ, Hou BH, Lalonde S, Takanaga H, et al. (2010). Sugar transporters for intercellular exchange and nutrition of pathogens. Nature 468: 527-532.

Chen LQ, Qu XQ, Hou BH, Sosso D, et al. (2012). Sucrose efflux mediated by SWEET proteins as a key step for phloem transport. Science 335: 207-211.

De Zutter JK, Levine KB, Deng D and Carruthers A (2013). Sequence determinants of GLUT1 oligomerization: analysis by homology-scanning mutagenesis. J. Biol. Chem. 288: 20734-20744.

Leto D and Saltiel AR (2012). Regulation of glucose transport by insulin: traffic control of GLUT4. Nat. Rev. Mol. Cell Biol. 13: 383-396.

Macheda ML, Williams ED, Best JD, Wlodek ME, et al. (2003). Expression and localisation of GLUT1 and GLUT12 glucose transporters in the pregnant and lactating rat mammary gland. Cell Tissue Res. 311: 91-97.

Nozaki S, Takeda T, Kitaura T, Takenaka N, et al. (2013). Akt2 regulates Rac1 activity in the insulin-dependent signaling pathway leading to GLUT4 translocation to the plasma membrane in skeletal muscle cells. Cell. Signal. 25: 1361-1371.

Saudek V (2012). Cystinosin, MPDU1, SWEETs and KDELR belong to a well-defined protein family with putative function of cargo receptors involved in vesicle trafficking. PLoS One 7: e30876.

Scheepers A, Joost HG and Schürmann A (2004). The glucose transporter families SGLT and GLUT: molecular basis of normal and aberrant function. JPEN J. Parenter. Enteral. Nutr. 28: 364-371.

Stuart CA, Howell ME, Zhang Y and Yin D (2009). Insulin-stimulated translocation of glucose transporter (GLUT) 12 parallels that of GLUT4 in normal muscle. J. Clin. Endocrinol. Metab. 94: 3535-3542. 
Tamura K, Peterson D, Peterson N, Stecher G, et al. (2011). MEGA5: molecular evolutionary genetics analysis using maximum likelihood, evolutionary distance, and maximum parsimony methods. Mol. Biol. Evol. 28: 2731-2739.

Thorens B and Mueckler M (2010). Glucose transporters in the 21st Century. Am. J. Physiol. Endocrinol. Metab. 298: E141-E145.

Walmsley AR, Barrett MP, Bringaud F and Gould GW (1998). Sugar transporters from bacteria, parasites and mammals: structure-activity relationships. Trends Biochem. Sci. 23: 476-481.

Xuan YH, Hu YB, Chen LQ, Sosso D, et al. (2013). Functional role of oligomerization for bacterial and plant SWEET sugar transporter family. Proc. Natl. Acad Sci. U S A 110: E3685-E3694.

Zhao FQ (2014). Biology of glucose transport in the mammary gland. J. Mammary Gland Biol. Neoplasia 19: 3-17.

Zhao FQ and Keating AF (2007). Functional properties and genomics of glucose transporters. Curr. Genomics. 8: 113-128. 